\title{
MicroRNA-208a directly targets Src kinase signaling inhibitor 1 to facilitate cell proliferation and invasion in non-small cell lung cancer
}

\author{
LI LIU $^{1,2}$, WUZHANG WANG ${ }^{2}$, SONG GAO $^{2}$ and XIUWEN WANG ${ }^{1}$ \\ ${ }^{1}$ Department of Chemotherapy, Cancer Center, Qilu Hospital of Shandong University, Jinan, Shandong 250012; \\ ${ }^{2}$ Minimally Invasive Interventional Diagnosis and Treatment Ward, Shandong Chest Hospital, \\ Jinan, Shandong 250013, P.R. China
}

Received June 1, 2018; Accepted February 22, 2019

DOI: $10.3892 / \mathrm{mmr} .2019 .10542$

\begin{abstract}
The abnormal expression of microRNAs (miRNAs/miRs) has a critical function in the formation and progression of non-small cell lung cancer (NSCLC). Therefore, understanding the association between NSCLC and dysregulated miRNAs may allow for the identification of novel diagnostic and therapeutic biomarkers for patients with this malignancy. Previous studies have validated miR-208a as a cancer-associated miRNA in multiple different types of human cancer, however, its expression pattern and precise function in NSCLC remains yet to be elucidated. Therefore, the aims of the present study were to measure miR-208a expression in NSCLC, investigate its specific functions in NSCLC and determine its exact regulatory mechanisms. Herein, the results demonstrated that miR-208a was significantly upregulated in NSCLC tissues and cell lines compared with that in adjacent non-cancerous tissues and a non-tumorigenic bronchial epithelium BEAS-2B cell line $(\mathrm{P}<0.05$, respectively). The high expression level of miR-208a exhibited an obvious association with Tumor-Node-Metastasis stage and lymph node metastasis. miR-208a silencing decreased the proliferative and invasive capacities of NSCLC cells. Notably, Src kinase signaling inhibitor 1 (SRCIN1) was verified as a potential direct target gene of miR-208a in NSCLC cells. Furthermore, SRCIN1 knockdown was able to rescue the miR-208a-mediated effects on NSCLC cells. In addition to this, silencing miR-208a expression inhibited the extracellular regulated kinase (ERK) signaling pathway in NSCLC. Overall, to the best of our knowledge, the present study is the first to provide evidence that miR-208a exerts
\end{abstract}

Correspondence to: Professor Xiuwen Wang, Department of Chemotherapy, Cancer Center, Qilu Hospital of Shandong University, 107 Wenhua West Road, Jinan, Shandong 250012, P.R. China E-mail: xiuwenwang12@sdu.edu.cn

Key words: microRNA-208a, Src kinase signaling inhibitor 1, non-small cell lung cancer, tumor-promoting microRNA oncogenic functions in the carcinogenesis and progression of NSCLC by directly targeting SRCIN1 and regulating the ERK pathway. Therefore, miR-208a may be developed as a potential target for treating patients with NSCLC.

\section{Introduction}

Lung cancer, one of the most prevalent human malignancies, is the leading cause of cancer-associated mortality globally (1). In total, 1.82 million novel lung cancer cases and 1.59 million mortalities occur per year globally, according to the data from GLOBOCAN 2012 (2). According to histological analysis, lung cancer may be divided into two predominant categories, namely small cell lung cancer and non-small cell lung cancer (NSCLC) (3). NSCLC accounts for $\sim 85 \%$ of all lung cancer cases (4). NSCLC includes three major subtypes, namely squamous-cell carcinoma, adenocarcinoma and large-cell carcinoma (4). Currently, surgery, radiotherapy and chemotherapy are the primary therapeutic techniques for patients with NSCLC (5). Despite advances in diagnosis and therapy in previous years, their clinical efficiency in patients with NSCLC remains unsatisfactory with a 5-year survival rate of only $15 \%(6,7)$. The late disease presentation, tumor heterogeneities within histological subtypes, cancer metastasis, high recurrence rates and the poor understanding of NSCLC pathogenesis are responsible for the poor prognosis of patients with NSCLC $(8,9)$. Therefore, the elucidation of the mechanisms underlying NSCLC genesis and development will help identify promising diagnostic indicators and therapeutic targets for the treatment of the malignancy.

MicroRNAs (miRNAs/miRs), which are 18-22 nucleotides in length, are an abundant class of endogenous, noncoding short RNA molecules (10). miRNAs may negatively regulate gene expression through the recognition and preferential binding to the 3'-untranslated regions (3'-UTRs) of their targets, which thus result in translation suppression and/or mRNA cleavage (11). miRNAs may modulate over one half of all gene expression of human proteins and may participate in the modulation of numerous cellular biological behaviors, including cell proliferation, survival, apoptosis, differentiation, metabolism and metastasis (12). Numerous deregulated miRNAs 
have been identified in various disorders, including human malignancies (13-15). The aberrant expression of miRNAs is strongly associated with the tumorigenesis and tumor development (16-18). miRNAs may function as tumor-suppressing or tumor-promoting miRNAs in different types of human cancer, depending on the biological functions of their target genes (19). As a consequence, research on cancer-associated miRNAs in NSCLC may benefit the identification of effective therapeutic methods for patients with NSCLC.

miR-208a is reportedly expressed abnormally in numerous human malignancies (20-22). The expression level and potential functions of miR-208a in NSCLC, however, remain unknown. Thus, the aims of the present study were to measure the expression level, clinical significance and detailed functions of miR-208a in NSCLC and the associated regulatory mechanism.

\section{Materials and methods}

Tissue specimens and ethical statement. A total of 52 primary NSCLC tissues (21 adenocarcinoma, 26 squamous cell carcinoma and 5 large cell neuroendocrine carcinoma) and adjacent non-cancerous tissues were obtained from Qilu Hospital of Shandong University (Shandong, China) between March 2014 and June 2016. All patients were treated with surgical resection. The present study included 28 males and 24 females, with an age range of 47-73 years (median age, 62 years). All participants had not undergone preoperative chemotherapy or radiotherapy. Patients who had been treated with preoperative chemotherapy or radiotherapy were excluded from this research. Tumor-Node-Metastasis (TNM) stage (23) was used for staging. Fresh surgical tissue samples were immediately frozen in liquid nitrogen and stored at $-80^{\circ} \mathrm{C}$ until used. The Ethics Committee of the Qilu Hospital of Shandong University ethically approved the present study. In addition, all patients provided written informed consent prior to the surgery.

Cell lines and culture conditions. In total, five NSCLC cell lines, including SK-MES-1, NCI-H522, NCI-H460, SPC-A1 and A-549, in addition to a non-tumorigenic bronchial epithelium BEAS-2B cell line were ordered from the Shanghai Institute of Biochemistry and Cell Biology (Shanghai, China).

LHC-9 medium (Gibco; Thermo Fisher Scientific, Inc., Waltham, MA, USA) containing $10 \%$ fetal bovine serum (FBS; Gibco; Thermo Fisher Scientific, Inc.) was utilized for the culture of the BEAS-2B cell line. Dulbecco's modified Eagle's medium (DMEM; Gibco; Thermo Fisher Scientific, Inc.) supplemented with $10 \%$ FBS was used to culture all the aforementioned NSCLC cell lines. All cells were grown at $37^{\circ} \mathrm{C}$ in a humidified condition supplied with $5 \% \mathrm{CO}_{2}$.

Reverse transcription-quantitative polymerase chain reaction $(R T-q P C R)$. TRIzol ${ }^{\circledR}$ (Invitrogen; Thermo Fisher Scientific, Inc.) was applied for the isolation of total RNA from tissue specimens or cultured cells as aforementioned. For the quantification of miR-208a, complementary DNA (cDNA) was prepared from total RNA with the TaqMan MicroRNA Reverse Transcription kit (Applied Biosystems; Thermo Fisher Scientific, Inc.) according to the manufacturer's protocol. The temperature protocol for reverse transcription was: $16^{\circ} \mathrm{C}$ for
$30 \mathrm{~min}, 42^{\circ} \mathrm{C}$ for $30 \mathrm{~min}$ and $85^{\circ} \mathrm{C}$ for $5 \mathrm{~min}$. Subsequently, cDNA was amplified by qPCR using the TaqMan MicroRNA PCR kit (Applied Biosystems; Thermo Fisher Scientific, Inc.) according to the manufacturer's protocol. The cycling conditions for qPCR were as follows: $50^{\circ} \mathrm{C}$ for $2 \mathrm{~min}, 95^{\circ} \mathrm{C}$ for $10 \mathrm{~min}$; 40 cycles of denaturation at $95^{\circ} \mathrm{C}$ for $15 \mathrm{sec}$; and annealing/extension at $60^{\circ} \mathrm{C}$ for $60 \mathrm{sec}$. For the detection of Src kinase signaling inhibitor 1 (SRCIN1) mRNA expression, reverse transcription was performed using the PrimeScript RT Reagent kit followed by qPCR using the SYBR Premix Ex Taq ${ }^{\mathrm{TM}}$ kit (both from Takara Biotechnology Co., Ltd., Dalian, China) according to the manufacturer's protocols. The temperature protocol for reverse transcription was: $37^{\circ} \mathrm{C}$ for $15 \mathrm{~min}$ and $85^{\circ} \mathrm{C}$ for $5 \mathrm{sec}$. The thermocycling conditions for qPCR were as follows: $95^{\circ} \mathrm{C}$ for $10 \mathrm{~min}$, followed by 40 cycles of $95^{\circ} \mathrm{C}$ for $15 \mathrm{sec}$ and $60^{\circ} \mathrm{C}$ for $1 \mathrm{~min}$. The expression levels of miR-208a and SRCIN1 mRNA were normalized to that of U6 snRNA and GAPDH, respectively. RT-qPCR was performed three times using an ABI7500 Real-Time PCR System (Applied Biosystems; Thermo Fisher Scientific, Inc.). The $2^{-\Delta \Delta \mathrm{Cq}}$ method (23) was used to analyze the relative miR-208a and SRCIN1 mRNA expression. The primers were designed as follows: miR-208a, 5'-GTCATCTAG AAAGCTTGATGCAGGAAAGAGCTTTGG-3' (forward) and 5'-TGACAGATCTCAGCTGA CATCCTCTAGGCT GGGGTT-3' (reverse); U6, 5'-GTGCTCGCTTCGGCAGCA CATAT-3' (forward) and 5'-AAAATATGGAACGCTTCA CGAA-3' (reverse); SRCIN1, 5'-GAACGGCTGCGCTATCT CAA-3' (forward) and 5'-GGATCTTCTCCACCGATTTC TCC-3' (reverse); and GAPDH, 5'-GCTGGCGCTGAGTAC GTCGTGGAGT-3' (forward) and 5'-CACAGTCTTCTGGGT GGCAGTGATGG-3' (reverse).

RNA oligonucleotide and cell transfection. The miR-208a inhibitor used to knockdown endogenous miR-208a expression was chemically synthesized by Shanghai GenePharma Co. Ltd. (Shanghai, China). The negative control (NC) miRNA inhibitor functioned as the control for miR-208a. Small interfering (si)RNA against the expression of SRCIN1 and its NC siRNA were purchased from Guangzhou RiboBio Co., Ltd. (Guangzhou, China). The SRCIN1 siRNA sequence was 5'-AAGCTGTGTCTGTTGAGGCTG-3' and the NC siRNA sequence was 5'-UUCUCCGAACGUGUCACGUTT-3'. For cell transfection, $\mathrm{H} 460$ and A549 cells were plated into 6 -well plates at a density of $6 \times 10^{5}$ cells per well. The transfection experiments were mediated using Lipofectamine 2000 (Invitrogen; Thermo Fisher Scientific, Inc.) based on the manufacturer's protocol. The quantity of siRNA transfected was $100 \mathrm{pmol}$. After $8 \mathrm{~h}$ incubation at $37^{\circ} \mathrm{C}$, transfected cells were washed with PBS (Gibco; Thermo Fisher Scientific, Inc.) and the culture medium was replaced with fresh DMEM containing $10 \%$ FBS.

Cell Counting Kit-8 (CCK-8) assay. A total of $24 \mathrm{~h}$ after transfection, the transfected H460 and A549 cells were harvested and subsequently inoculated into the 96-well plates at an initial density of 3,000 cells/well. The cells were then incubated at $37^{\circ} \mathrm{C}$ in a humidified incubator with $5 \% \mathrm{CO}_{2}$ for $0,24,48$ and $72 \mathrm{~h}$. A CCK-8 assay was applied to measure cellular proliferation at each time point. The cells were then 
incubated with a total of $10 \mu \mathrm{lCCK}-8$ assay solution (Dojindo Molecular Technologies, Inc., Kumamoto, Japan) at $37^{\circ} \mathrm{C}$ for additional $2 \mathrm{~h}$. The optical density at $450 \mathrm{~nm}$ wavelength was read with a microplate reader (BioTek Instruments, Inc., Winooski, VT, USA).

Matrigel invasion assay. A Matrigel invasion assay was adopted to evaluate the cellular invasive ability using a Matrigel pre-coated 24-well Boyden chamber (BD Biosciences, Franklin Lakes, NJ, USA). At 48 h post-transfection, the H460 and A549 cells were harvested, washed with phosphate buffer solution and suspended in FBS-free DMEM. A total of $5 \times 10^{4}$ cells were seeded on the upper chambers and allowed to invade the reverse side of the chamber under chemoattractant conditions with $10 \%$ FBS medium in the lower chambers. After $24 \mathrm{~h}$ of culture at $37^{\circ} \mathrm{C}$, the non-invaded cells that remained on the upper surface of the upper chambers were removed gently using a cotton swab. The invaded cells were fixed using $100 \%$ methanol at room temperature for $30 \mathrm{~min}$ and stained with $0.1 \%$ crystal violet at room temperature for $30 \mathrm{~min}$. Finally, the invasive ability was assessed by counting the number of invaded cells in five randomly selected visual fields under an IX71 inverted light microscope (x200 magnification; Olympus Corporation, Tokyo, Japan).

miR-208a target prediction and luciferase reporter assay. The following online miRNA target prediction algorithms, TargetScan (24) (http://www.targetscan.org/index. html) and miRanda (25) (http://www. microrna.org/microrna/), were utilized to identify the putative target genes of miR-208a. Luciferase plasmids were chemically synthesized by Shanghai GenePharma Co., Ltd. The 3'-UTR segments of the SRCIN1 gene containing the wild-type or mutant miR-208a binding sites were inserted into pMIR-GLO ${ }^{\mathrm{TM}}$ luciferase vector to generate the wild-type luciferase plasmid (pMIR-SRCIN1-Wt-3'-UTR) or mutant luciferase plasmid (pMIR-SRCIN1-Mut-3'-UTR). $\mathrm{H} 460$ and A549 cells were inoculated into the 24-well plates at a density of 60 to $70 \%$ confluence. miR-208a inhibitor or NC inhibitor were cotransfected with pMIR-SRCIN1-Wt-3'-UTR or pMIR-SRCIN1-Mut-3'-UTR into the cells using Lipofectamine $^{\mathrm{TM}} 2000$ reagent. Subsequent to incubation at $37^{\circ} \mathrm{C}$ for $48 \mathrm{~h}$, transfected cells were harvested and subjected to the quantification of luciferase activity using a dual-luciferase reporter assay system (Promega Corporation, Madison, WI, USA). Luciferase activity was normalized relative to that of the Renilla luciferase activity.

Western blot analysis. Total protein was isolated from tissue samples or cultured H460 and A549 cells using radioimmunoprecipitation assay lysis buffer (Sigma-Aldrich; Merck KGaA, Darmstadt, Germany) supplemented with protease inhibitors (Roche Diagnostics, Basel, Switzerland). A bicinchoninic acid kit (Beyotime Institute of Biotechnology, Shanghai, China) was adopted to measure the concentration of the total protein extracts, according to the manufacturer's protocol. Equal amounts of proteins $(30 \mu \mathrm{g})$ were loaded, separated by $10 \%$ SDS-PAGE and then transferred to polyvinylidene difluoride membranes (EMD Millipore, Billerica, MA, USA). Following blocking in $5 \%$ non-fat milk at room temperature for $2 \mathrm{~h}$, the membranes were incubated overnight at $4^{\circ} \mathrm{C}$ with primary antibodies against SRCIN1 (cat. no. 3757; Cell Signaling Technology, Inc., Danvers, MA, USA), extracellular regulated kinase (ERK; cat. no. sc-514302; Santa Cruz Biotechnology, Inc., Dallas, TX, USA), phosphorylated (p-)ERK (cat. no. sc-7383; Santa Cruz Biotechnology, Inc.) or GAPDH (cat. no. sc-51907; Santa Cruz Biotechnology, Inc.). All primary antibodies were used at a dilution of 1:1,000. Subsequently, the membranes were rinsed with Tris-buffered saline containing $0.1 \%$ Tween-20 (TBST) for three times and probed using a goat anti-mouse IgG-HRP secondary antibody conjugated with horseradish peroxidase (1:5,000 dilution; cat. no. sc-2005; Santa Cruz Biotechnology, Inc.) at room temperature for $1 \mathrm{~h}$. An enhanced chemiluminescence immunoblot detection system (Pierce; Thermo Fisher Scientific, Inc.) was applied to visualize the protein signals. Relative protein expression was analyzed using Quantity One software (version 4.62; Bio-Rad Laboratories, Inc., Hercules, CA, USA) and presented as the density ratio compared with GAPDH.

Statistical analysis. All data were presented as the mean \pm standard deviation and analyzed using SPSS software version 18.0 (SPSS, Inc., Chicago, IL, USA). A Student's t-test and one-way analysis of variance followed by a Tukey or Dunnett's test were used to compare the differences between two groups and multiple groups, respectively. Spearman's correlation analysis was performed to examine the association between miR-208a and SRCIN1 in NSCLC tissues. $\mathrm{P}<0.05$ was considered to indicate a statistically significant difference.

\section{Results}

miR-208a is overexpressed in NSCLC tissue specimens and cell lines. To investigate the expression profile of miR-208a in NSCLC, RT-qPCR was utilized for the determination of miRNA expression levels in NSCLC tissues and adjacent non-cancerous tissues. The expression levels of miR-208a were significantly upregulated in NSCLC tissues compared with that in adjacent non-cancerous tissues $(\mathrm{P}<0.05$; Fig. 1A). Subsequently, miR-208a expression was detected in five human NSCLC cell lines, including SK-MES-1, H522, H460, SPC-A1 and A-549. Fig. 1B reveals that the miR-208a expression levels were significantly higher in all NSCLC cell lines compared with that in the non-tumorigenic bronchial epithelium BEAS-2B cell line $(\mathrm{P}<0.05)$. To investigate the clinical value of miR-208a dysregulation in patients with NSCLC, all patients with NSCLC were divided into miR-208a high/low expression groups based on median expression of miR-208a (1.83). As presented in Table I, high miR-208a expression was significantly associated with Tumor-Node-Metastasis (TNM) stage $(\mathrm{P}=0.026)$ and lymph node metastasis $(\mathrm{P}=0.012)$. These results suggest that miR-208a may serve an important function in the development of NSCLC.

miR-208a inhibition attenuates the proliferation and invasion of NSCLC cells. To clarify the biological function of miR-208a in NSCLC progression, a miR-208a inhibitor was introduced into $\mathrm{H} 460$ and A549 cells, which expressed a relatively high level of miR-208 among the five NSCLC cell lines, to knock down endogenous miR-208a expression. miR-208a revealed a significant decrease in H460 and A549 cells following 
Table I. Association between miR-208a expression and the clinicopathological features of patients with non-small cell lung cancer.

\begin{tabular}{|c|c|c|c|c|}
\hline \multirow[b]{2}{*}{ Clinicopathological features } & \multirow[b]{2}{*}{ Cases } & \multicolumn{2}{|c|}{$\begin{array}{c}\text { miR-208a } \\
\text { expression } \\
\text { group }\end{array}$} & \multirow[b]{2}{*}{ P-value } \\
\hline & & High & Low & \\
\hline Sex & & & & 0.266 \\
\hline Male & 28 & 12 & 16 & \\
\hline Female & 24 & 14 & 10 & \\
\hline Age & & & & 0.578 \\
\hline$<60$ years & 28 & 15 & 13 & \\
\hline$\geq 60$ years & 24 & 11 & 13 & \\
\hline Tumor size & & & & 0.779 \\
\hline$<5 \mathrm{~cm}$ & 22 & 10 & 12 & \\
\hline$\geq 5 \mathrm{~cm}$ & 30 & 16 & 14 & \\
\hline Smoking history & & & & 0.760 \\
\hline$<10$ years & 15 & 7 & 8 & \\
\hline$\geq 10$ years & 37 & 19 & 18 & \\
\hline Tumor differentiation & & & & 0.780 \\
\hline I-II & 23 & 11 & 12 & \\
\hline III-IV & 29 & 15 & 14 & \\
\hline Tumor-Node-Metastasis stage & & & & 0.026 \\
\hline I-II & 24 & 8 & 16 & \\
\hline III-IV & 28 & 18 & 10 & \\
\hline Lymph node metastasis & & & & 0.012 \\
\hline Negative & 27 & 9 & 18 & \\
\hline Positive & 25 & 17 & 8 & \\
\hline
\end{tabular}

Tumor differentiation I, well-differentiated; II, moderately differentiated; III, poorly differentiated; IV, undifferentiated. miR, microRNA.

miR-208a knockdown compared with the NC inhibitor group ( $\mathrm{P}<0.05$; Fig. $2 \mathrm{~A})$. A CCK-8 assay was performed to determine whether NSCLC cell proliferation may be influenced by miR-208a. As expected, cell proliferation in H460 and A549 cells was significantly decreased with miR-208a inhibitor transfection compared with the NC inhibitor group $(\mathrm{P}<0.05$; Fig. 2B). The effect of silencing miR-208a expression on cell invasion capacity in NSCLC was evaluated using a Matrigel invasion assay. The results revealed that the numbers of invaded cells were significantly reduced in miR-208a inhibitor-transfected $\mathrm{H} 460$ and A549 cells compared with the NC inhibitor groups $(\mathrm{P}<0.05$; Fig. $2 \mathrm{C})$. These results suggest that miR-208a may suppress the growth of a tumor in NSCLC progression.

miR-208a directly targets SRCIN1 in NSCLC. To investigate the mechanism by which miR-208a regulates the malignant phenotypes in NSCLC, bioinformatics analysis was performed to predict the putative target genes with complementary sites of miR-208a in their 3'-UTR. SRCIN1, which has been reported to be implicated in NSCLC occurrence and development (26-28), was predicted as a major candidate of miR-208a (Fig. 3A). To verify whether miR-208a may directly interact with the 3'-UTR of SRCIN1, a luciferase reporter assay was performed in $\mathrm{H} 460$ and A549 cells that were co-transfected with miR-208a inhibitor or NC inhibitor and pMIR-SRCIN1-Wt-3'-UTR or pMIR-SRCIN1-Mut-3'-UTR. miR-208a inhibition induced a significant increase in luciferase activity for pMIR-SRCIN1-Wt-3'-UTR in H460 and A549 cells compared with the NC inhibitor control group $(\mathrm{P}<0.05)$. This effect, however, was abrogated when harboring mutations were present at the predicted SRCIN1 3'-UTR-binding sequences for miR-208a (Fig. 3B and C). RT-qPCR analysis and western blot analysis were also performed to determine whether miR-208a regulates SRCIN1 expression in NSCLC cells. The expression levels of SRCIN1 mRNA $(\mathrm{P}<0.05)$ and protein $(\mathrm{P}<0.05)$ in miR-208a inhibitor-transfected $\mathrm{H} 460$ and A549 cells were significantly upregulated in comparison with those in NC inhibitor-transfected cells (Fig. 3D and E).

SRCIN1 mRNA expression was then detected in 52 primary NSCLC tissues to further investigate the association between miR-208a and SRCIN1 in NSCLC. The results revealed that NSCLC tissues exhibited significantly lower SRCIN1 mRNA expression levels in comparison with those of the adjacent non-cancerous tissues $(\mathrm{P}<0.05$; Fig. $3 \mathrm{~F})$. Furthermore, an significant inverse expression correlation between miR-208a and SRCIN1 mRNA levels in NSCLC tissues was identified through Spearman's correlation analysis $(\mathrm{r}=-0.6273, \mathrm{P}<0.001$; Fig. 3G). Collectively, SRCIN1 was generally revealed to be a direct target of miR-208a in NSCLC.

Knockdown of SRCIN1 expression partially reverses the influence of miR-208a inhibitor on NSCLC cells. A number of rescue experiments were applied to determine whether the oncogenic function of miR-208a on NSCLC cell progression is mediated by SRCIN1 upregulation. SRCIN1 siRNA or NC siRNA along with miR-208a inhibitor were transfected into H460 and A549 cells. The co-transfection of SRCIN1 siRNA significantly abrogated the miR-208a inhibitor-mediated upregulation of SRCIN1 in H460 and A549 cells, as demonstrated by western blot analysis $(\mathrm{P}<0.05$; Fig. 4A). In addition, the knockdown of SRCIN1 expression in H460 and A549 cells transfected with miR-208a inhibitor significantly rescued the inhibition of cell proliferation $(\mathrm{P}<0.05$; Fig. 4B) and invasion $(\mathrm{P}<0.05$; Fig. 4C) caused by miR-208a underexpression. On the basis of the aforementioned results, it was concluded that the tumor-promoting functions of miR-208a in NSCLC cells are, at least in part, attributable to SRCIN1 regulation.

miR-208a inhibition reduces the activity of the ERK signaling pathway in NSCLC. One previous study has demonstrated that SRCIN1 regulates the ERK pathway in lung cancer (26). Thus, the present study aimed to determine whether miR-208a may regulate the ERK signaling pathway in NSCLC. Western blot analysis was utilized to measure the expression of ERK and p-ERK proteins in H460 and A549 cells subsequent to co-transfection with miR-208a inhibitor and SRCIN1 siRNA or NC siRNA. As expected, miR-208a inhibition was revealed to reduce p-ERK expression in H460 and A549 cells (Fig. 5), whereas the total ERK protein expression was unaffected. In addition, the protein expression of p-ERK in H460 and A549 

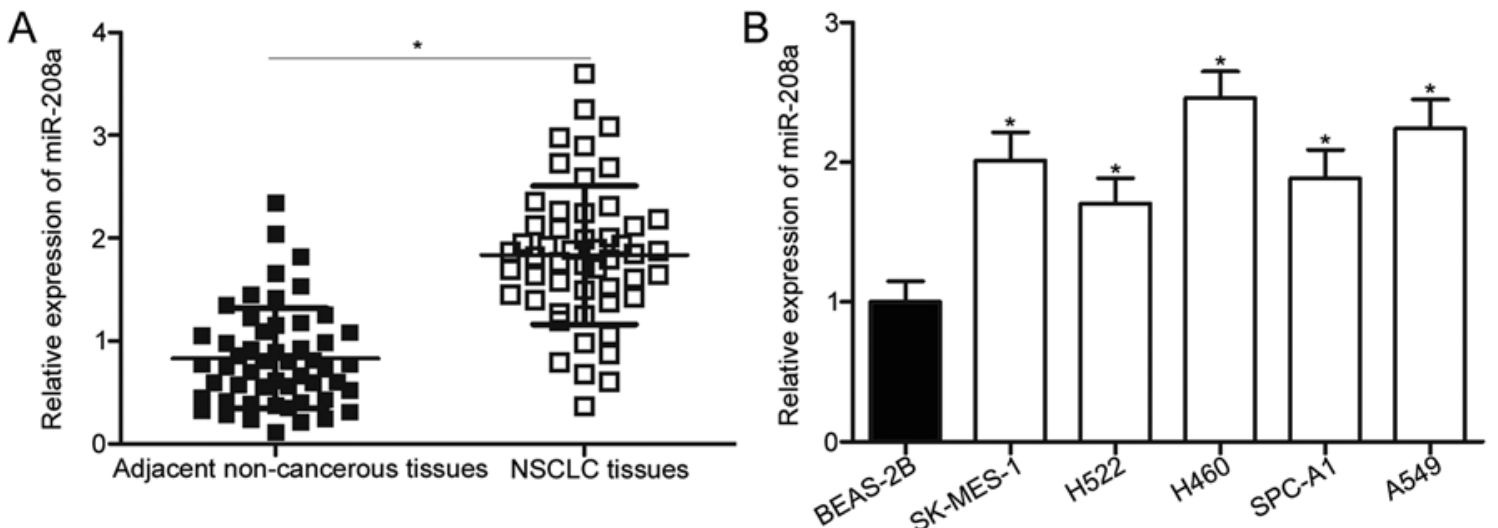

Figure 1. miR-208a is upregulated in NSCLC tissues and cell lines. (A) Relative miR-208a expression levels in 52 primary NSCLC tissues and adjacent non-cancerous tissues were detected using RT-qPCR. ${ }^{*} \mathrm{P}<0.05$ with comparisons shown by lines. (B) RT-qPCR analysis of miR-208a expression levels in five NSCLC cell lines (SK-MES-1, H522, H460, SPC-A1 and A549) and a non-tumorigenic bronchial epithelium BEAS-2B cell line. "P<0.05 vs. the BEAS-2B cell line. miR, microRNA; NSCLC, non-small cell lung cancer; RT-qPCR, reverse transcription-quantitative polymerase chain reaction.
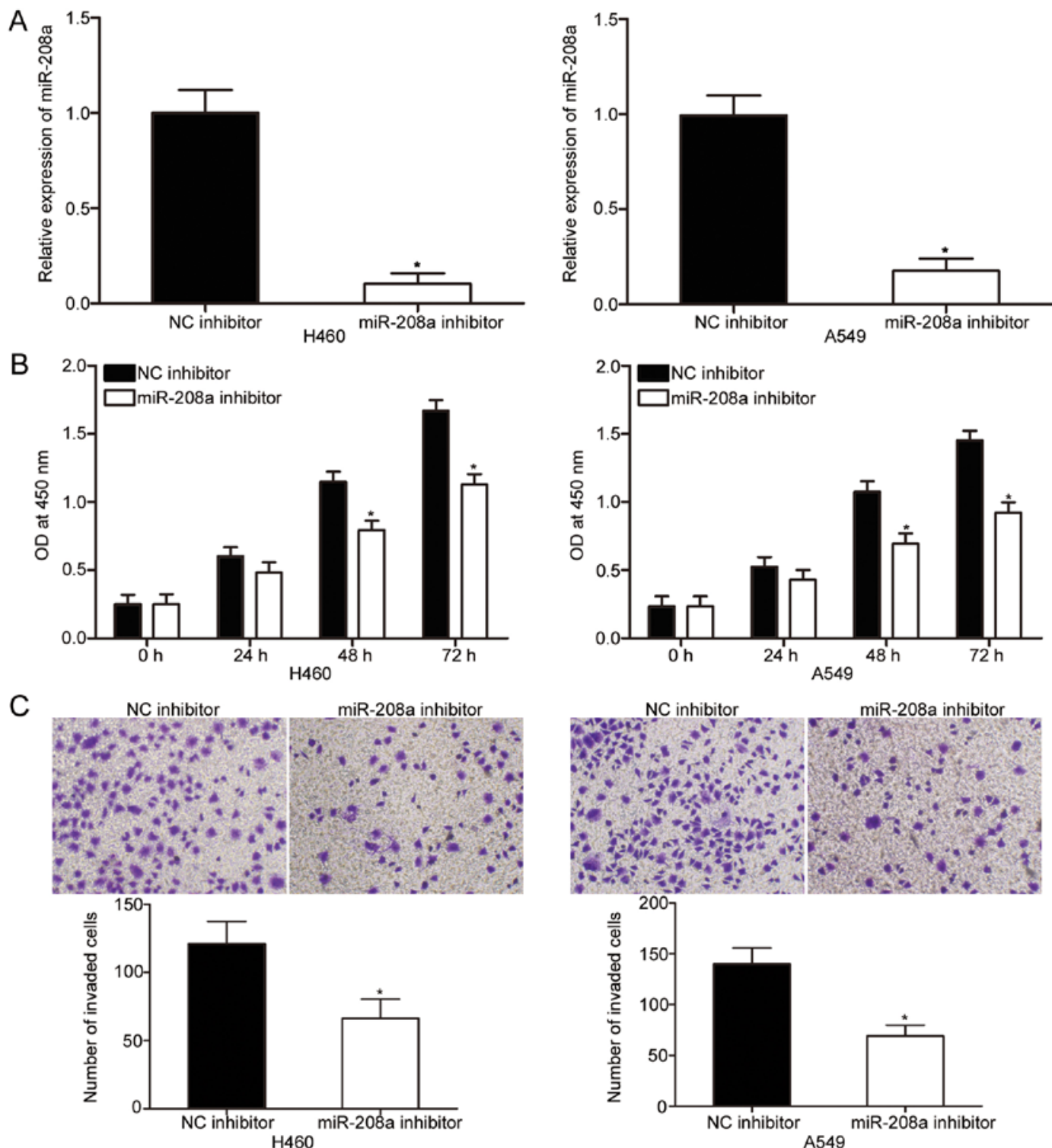

Figure 2. miR-208a downregulation inhibits the proliferation and invasion of H460 and A549 cells. (A) H460 and A549 cells were transfected with miR-208a inhibitor or an NC inhibitor followed by reverse transcription-quantitative polymerase chain reaction analysis of the miRNA levels in the differently treated cells. ${ }^{*} \mathrm{P}<0.05$ vs. the NC inhibitor group. (B) Cell Counting Kit- 8 assays were performed to determine the proliferation of H460 and A549 cells following transfection with an miR-208a inhibitor or NC inhibitor. ${ }^{*} \mathrm{P}<0.05$ vs. the $\mathrm{NC}$ inhibitor group. (C) Cell invasion capacity was evaluated in $\mathrm{H} 460$ and A549 cells transfected with miR-208a inhibitor or NC inhibitor. " $\mathrm{P}<0.05$ vs. the NC inhibitor group. miR/miRNA, microRNA; NC, negative control; OD, optical density. 
A
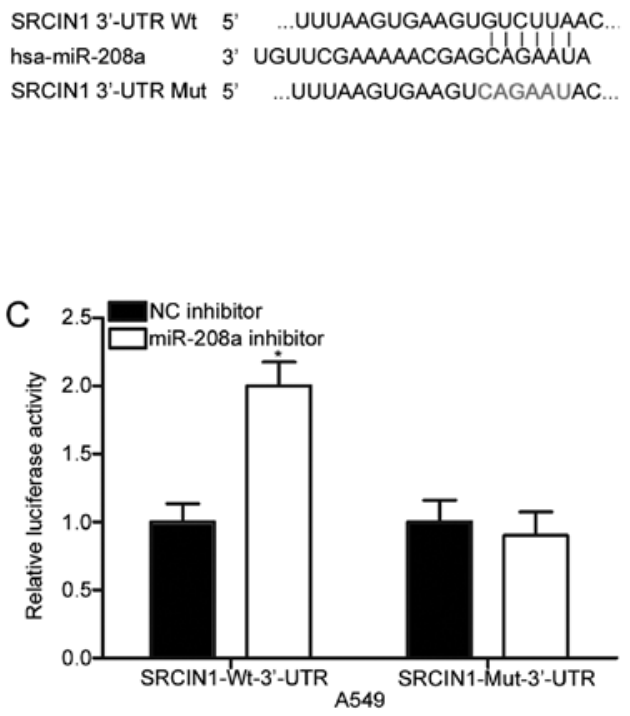

$\mathrm{E}$

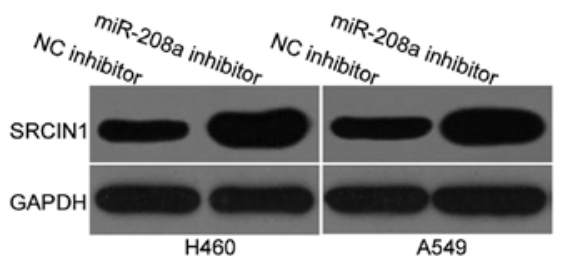

$\mathrm{F}$

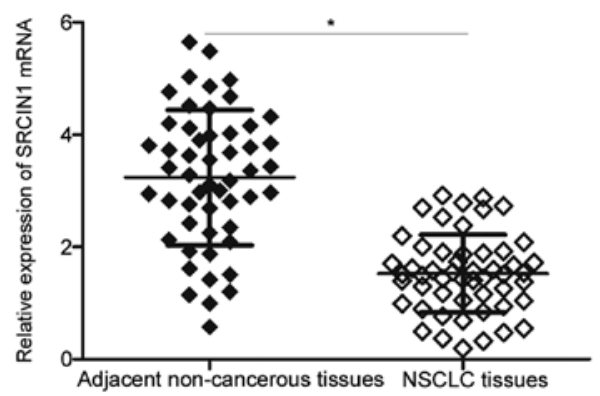

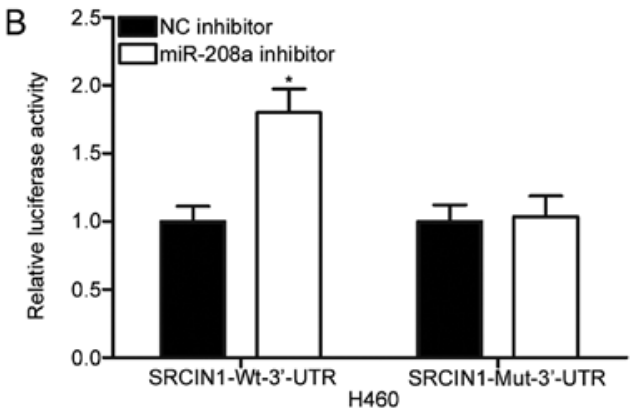
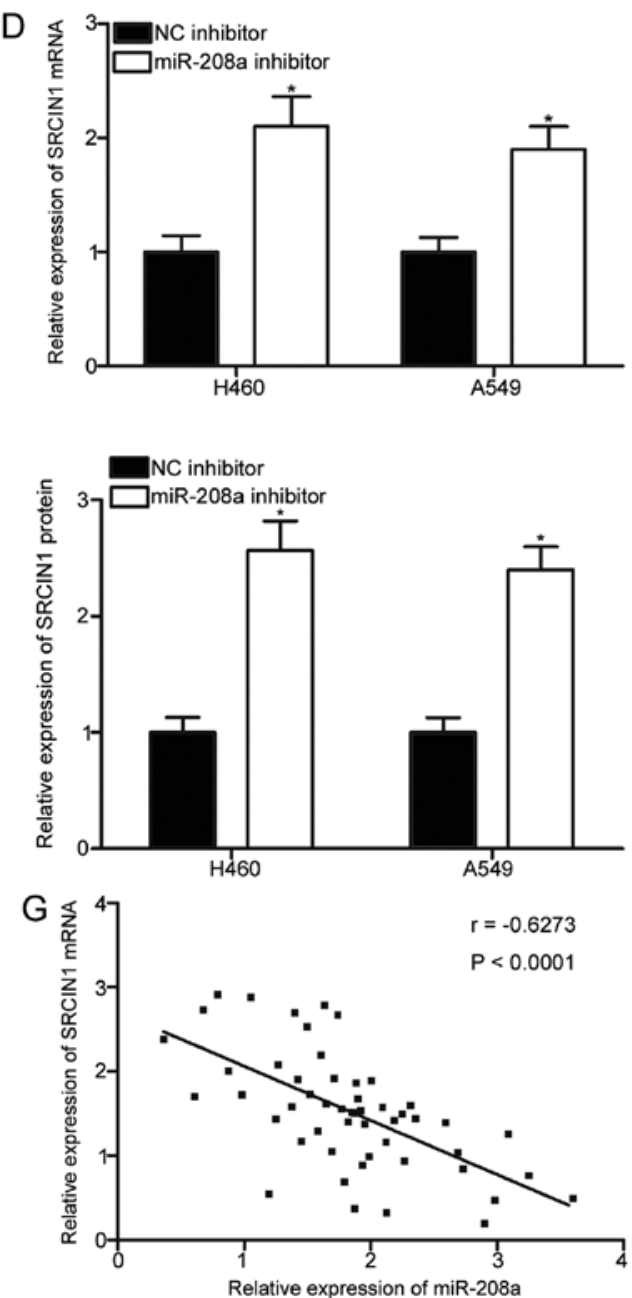

Figure 3. miR-208a directly targets SRCIN1 by directly binding to its 3'-UTR. (A) Putative Wt and Mut miR-208 binding sites in the 3'-UTR of SRCIN1. A luciferase reporter assay was performed to detect luciferase activity in (B) $\mathrm{H} 460$ and (C) A549 cells that were co-transfected with miR-208a inhibitor or NC inhibitor and pMIR-SRCIN1-Wt-3'-UTR or pMIR-SRCIN1-Mut-3'-UTR. " $\mathrm{P}<0.05$ vs. the NC inhibitor group. H460 and A549 cells were transfected with a miR-208a inhibitor or NC inhibitor. RT-qPCR and western blot analysis were performed to measure (D) SRCIN1 mRNA and (E) protein expression levels in the indicated cell lines. ${ }^{*} \mathrm{P}<0.05$ vs. the NC inhibitor group. (F) Expression levels of SRCIN1 mRNA in 52 primary NSCLC tissues and adjacent non-cancerous tissues were examined using RT-qPCR. "P<0.05 with comparisons shown by lines. (G) Spearman's correlation analysis was performed to assess the association between miR-208a and SRCIN1 mRNA in NSCLC tissues. miR, microRNA; SRCIN1, Src kinase signaling inhibitor 1; 3'-UTR, 3'untranslated region; Wt, wild type; Mut, mutant type; NC, negative control; NSCLC, non-small cell lung cancer; RT-qPCR, reverse transcription-quantitative polymerase chain reaction.

cells were recovered following co-transfection with SRCIN1 siRNA. These results suggest that miR-208a downregulation deactivates the ERK signaling pathway via the regulation of SRCIN1.

\section{Discussion}

Abnormal miRNA expression has been demonstrated to serve critical functions in NSCLC formation and progression $(29,30)$.
Therefore, understanding the association between NSCLC and abnormally expressed miRNAs may allow for the identification of numerous novel diagnostic and therapeutic biomarkers for patients with NSCLC. The present study is the first to present data, to the best of our knowledge, characterizing the expression profile, specific functions and associated mechanisms underlying miR-208a in NSCLC. miR-208a was notably upregulated in NSCLC tissues and cell lines compared with the non-cancerous controls. High expression levels of miR-208a 

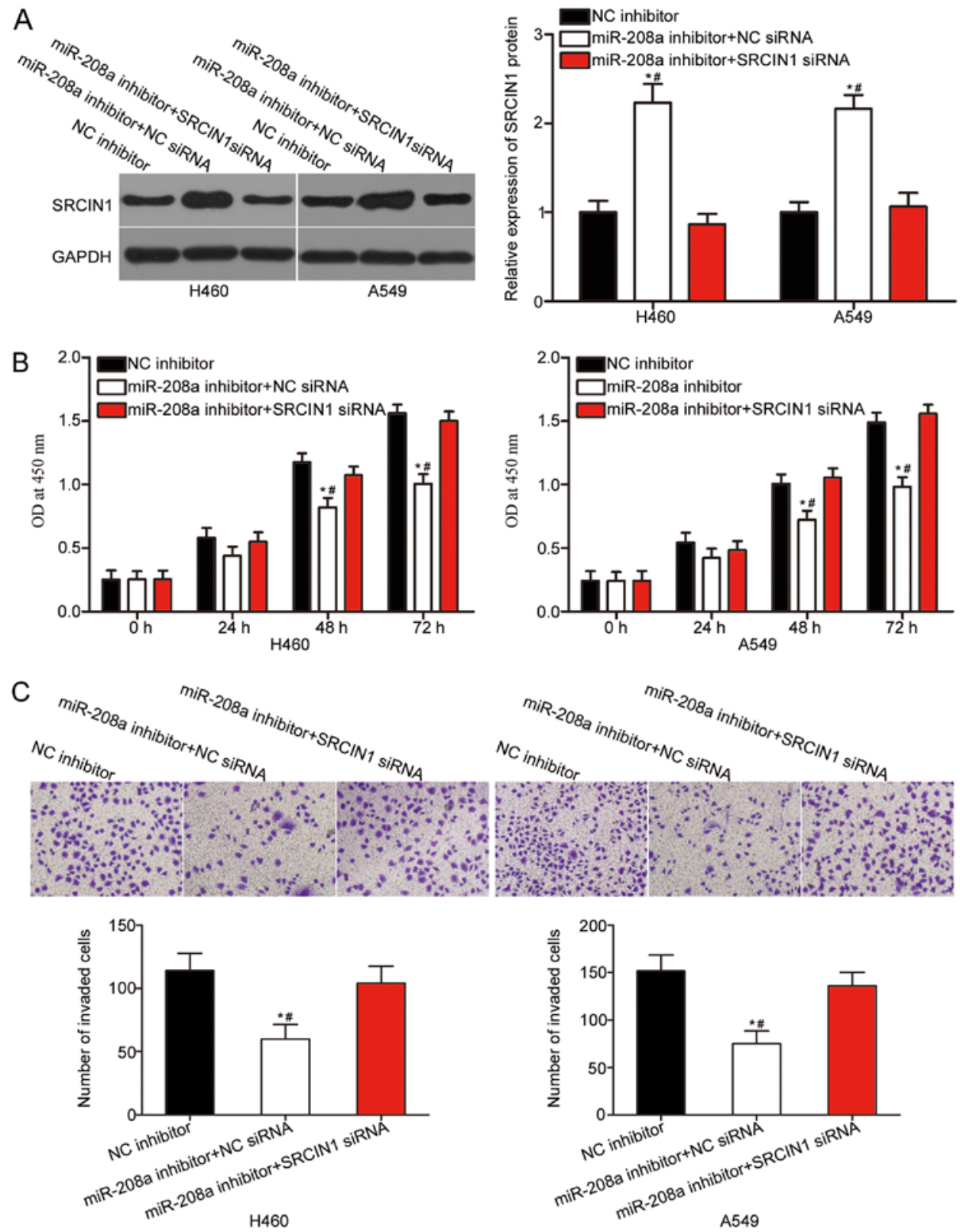

Figure 4. Knockdown of SRCIN1 expression partially inhibited the effects of miR-208a downregulation on the proliferation and invasion of H460 and A549 cells. H460 and A549 cells were co-transfected with an miR-208a inhibitor and SRCIN1 siRNA or NC siRNA. (A) Western blot analysis was performed to examine SRCIN1 protein expression levels in the differently treated cells $72 \mathrm{~h}$ following transfection. ${ }^{*} \mathrm{P}<0.05$ vs. the NC siRNA group. ${ }^{~} \mathrm{P}<0.05$ vs. the miR-208a inhibitor+SRCIN1 siRNA group. Cell Counting Kit-8 and Matrigel invasion assays were performed to determine (B) cell proliferation and (C) invasion, respectively, in the differently treated cells. " $\mathrm{P}<0.05$ vs. the NC siRNA group. ${ }^{*} \mathrm{P}<0.05$ vs. the miR-208a inhibitor+SRCIN1 siRNA group. miR, microRNA; SRCIN1, Src kinase signaling inhibitor 1; NC, negative control; siRNA, small interfering RNA; OD, optical density.
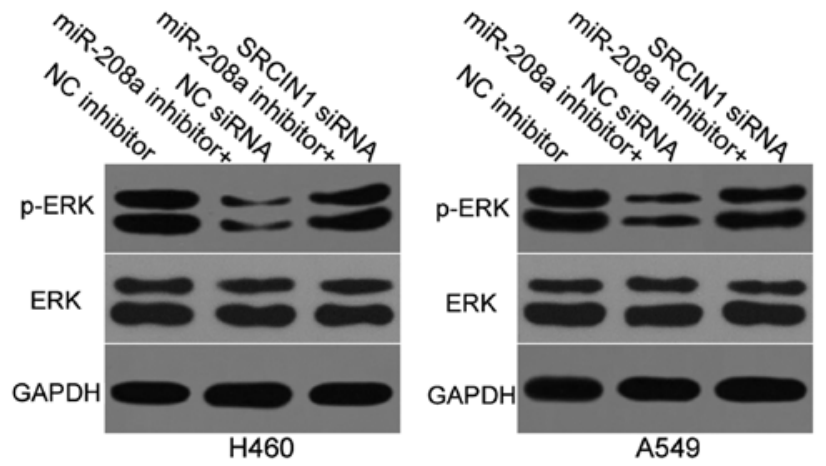

Figure 5. miR-208a affects the ERK signaling pathway in NSCLC. Western blot analysis detected the expression of ERK and p-ERK proteins in H460 and A549 cells following co-transfection with miR-208a inhibitor and SRCIN1 siRNA or NC siRNA. miR, microRNA; SRCIN1, Src kinase signaling inhibitor 1; NC, negative control; siRNA, small interfering RNA; ERK, extracellular regulated kinase; p-, phosphorylated. 
were significantly associated with TNM stage and lymph node metastasis in NSCLC. Meanwhile, miR-208a downregulation attenuated the proliferation and invasion of NSCLC cells. SRCIN1 was validated as a direct target of miR-208a in NSCLC. Silencing SRCIN1 expression partially reversed the oncogenic effects of miR-208a on NSCLC cell proliferation and invasion. Inhibition of miR-208a reduced the activity of the ERK signaling pathway in NSCLC through the regulation of SRCIN1. These results suggest that miR-208a may exert tumor-promoting functions in NSCLC and may be further developed as a novel target in treating patients with NSCLC.

miR-208a has been associated with multiple types of human cancer. For instance, miR-208a has been revealed to be upregulated in hepatocellular carcinoma. Inhibition of miR-208a inhibited hepatocellular carcinoma cell proliferation and invasion in vitro and decreased tumorigenesis in vivo (20). Li et al (21) reported that miR-208a was highly expressed in oesophageal squamous cell carcinoma tissues and cell lines. miR-208a upregulation facilitated the cell proliferation, tumorigenicity and cell cycle progression of oesophageal squamous cell carcinoma. Yin et al (22) also revealed that miR-208a was overexpressed in gastric cancer. miR-208a overexpression attenuated gastric cancer cell apoptosis and induced tumor growth in vivo. Liu et al (31) revealed that the ectopic expression of miR-208a promoted the cell migration, invasion and epithelial-mesenchymal transition of pancreatic cancer. Accordingly, miR-208a serves an oncogenic function in tumorigenesis and tumor development and may be developed as a potential target in the therapy of these specific tumor types.

A number of target miR-208a's have been identified, including AT-rich interactive domain-containing protein 1 in hepatocellular carcinoma (20), SRY-Box 6 in oesophageal squamous cell carcinoma (21) and programmed cell death 4 in gastric cancer (22). SRCIN1, also known as p140 cas-associated protein, has been demonstrated to be a direct target gene of miR-208a in NSCLC. The gene contains two coiled-coil domains, two proline-rich regions and two regions of highly charged amino acids (32). SRCIN was previously reported to be decreased in multiple human malignancy types, including liver cancer (33), cutaneous squamous cell carcinoma (34), breast cancer (35) and osteosarcoma (36). SRCIN1 was revealed to serve an inhibitory function in tumorigenesis and tumor development. For instance, SRCIN1 restoration repressed cell proliferation, colony formation, invasion and epithelial-mesenchymal transition in osteosarcoma (36). Resumption expression of SRCIN1 prohibited the proliferation and epithelial-mesenchymal transition in hepatocellular carcinoma (33). Ectopic expression of SRCIN1 in cutaneous squamous cell carcinoma suppressed the proliferative and migratory abilities of the cells (34). In the present study, it was demonstrated that miR-208a silencing deactivated the ERK signaling pathway via the regulation of SRCIN1. The ERK signaling pathway serves crucial functions in the occurrence and development of NSCLC, and is implicated in the regulation of aggressive phenotypes of NSCLC cells (37-39). These results suggest that restoring SRCIN1 expression may be adopted as a novel therapeutic strategy for anti-tumor therapy.

SRCIN1 has been demonstrated to be regulated by multiple miRNAs in NSCLC. For example, Cao et al (26) revealed that miR-150 targeted SRCIN1 to promote the proliferation and migration of NSCLC cells. Ye et al (27) reported that miR-211 induced cell growth in NSCLC through the negative regulation of SRCIN1. Gao et al (28) also identified that miR-873 increased the cell proliferation and migration of NSCLC cells via a SRCIN1 blockade. Zhang et al (40) indicated that miR-150 enhanced cell growth in vitro and in vivo by directly targeting SRCIN1. The present study demonstrated that the downregulation of miR-208a reduced NSCLC cell proliferation and invasion through SRCIN1 upregulation. These results suggest that the miRNA/SRCIN1 pathway may have certain clinical applications in the management of patients with NSCLC.

In summary, miR-208a was frequently overexpressed in NSCLC, and increased miR-208a expression was associated with TNM stage and lymph node metastasis. miR-208a may function as an oncogene by directly targeting SRCIN1 in NSCLC. The miR-208a/SRCIN1 axis may be used in miRNA-based therapy for the treatment of patients with NSCLC. However, the association between miR-208 and the overall survival or disease-free survival of patients with NSCLC was unexplored in the present study. It was a limitation of the present study, and survival information will be collected in order to resolve this limitation in the near future.

\section{Acknowledgements}

Not applicable.

Funding

No funding was received.

\section{Availability of data and materials}

The datasets used and/or analyzed during the present study are available from the corresponding author on reasonable request.

\section{Authors' contributions}

XW designed the present study and analyzed the data. LL performed RT-qPCR and the CCK-8 assay. Matrigel invasion assay and luciferase reporter assay were performed by WW. SG conducted western blotting. All authors have read and approved the final manuscript.

\section{Ethics approval and consent to participate}

The Ethics Committee of the Qilu Hospital of Shandong University ethically approved the present study. All patients provided written informed consent prior to the operation.

\section{Patient consent for publication}

All patients provided written informed consent prior to the operation. All patients provided consent for publication.

\section{Competing interests}

The authors declare that they have no competing interests. 


\section{References}

1. Siegel RL, Miller KD and Jemal A: Cancer statistics, 2016. CA Cancer J Clin 66: 7-30, 2016.

2. Ferlay J, Soerjomataram I, Dikshit R, Eser S, Mathers C, Rebelo M, Parkin DM, Forman D and Bray F: Cancer incidence and mortality worldwide: Sources, methods and major patterns in GLOBOCAN 2012. Int J Cancer 136: E359-E386, 2015.

3. Latimer KM: Lung cancer: Clinical presentation and diagnosis. FP Essent 464: 23-26, 2018.

4. Gadgeel SM: New targets in non-small cell lung cancer. Curr Oncol Rep 15: 411-423, 2013.

5. Mott TF: Lung cancer: Management. FP Essent 464: 27-30, 2018

6. Yu JL, Simmons C, Victor JC, Han D, Hogeveen S, Leighl N and Verma S: Impact of new chemotherapeutic and targeted agents on survival in stage IV non-small cell lung cancer. Oncologist 16 1307-1315, 2011

7. Parums DV: Current status of targeted therapy in non-small cell lung cancer. Drugs Today (Barc) 50: 503-525, 2014.

8. Simon J: Technology radiation technology targets tumors. Surgical precision without the incision. S D Med 67: 362, 2014.

9. Miller YE: Pathogenesis of lung cancer: 100 year report. Am J Respir Cell Mol Biol 33: 216-223, 2005.

10. Diniz GP and Wang DZ: Regulation of skeletal muscle by microRNAs. Compr Physiol 6: 1279-1294, 2016.

11. Bao W, Greenwold MJ and Sawyer RH: Expressed miRNAs target feather related mRNAs involved in cell signaling, cell adhesion and structure during chicken epidermal development. Gene 591: 393-402, 2016.

12. Bartel DP: MicroRNAs: Genomics, biogenesis, mechanism, and function. Cell 116: 281-297, 2004.

13. Croce CM and Calin GA: miRNAs, cancer, and stem cell division. Cell 122: 6-7, 2005.

14. Lin S and Gregory RI: MicroRNA biogenesis pathways in cancer. Nat Rev Cancer 15: 321-333, 2015.

15. Hata A and Lieberman J: Dysregulation of microRNA biogenesis and gene silencing in cancer. Sci Signal 8: re3, 2015.

16. Zhang A, Lakshmanan J, Motameni A and Harbrecht BG: MicroRNA-203 suppresses proliferation in liver cancer associated with PIK3CA, p38 MAPK, c-Jun, and GSK3 signaling. Mol Cell Biochem 441: 89-98, 2018.

17. Zhang Z, Yang $Y$ and Zhang X: MiR-770 inhibits tumorigenesis and EMT by targeting JMJD6 and regulating WNT/ $\beta$-catenin pathway in non-small cell lung cancer. Life Sci 188: 163-171, 2017.

18. Zhang X, Ma X, An H, Xu C, Cao W, Yuan W and Ma J: Upregulation of microRNA-125b by G-CSF promotes metastasis in colorectal cancer. Oncotarget 8: 50642-50654, 2017.

19. Volinia S, Calin GA, Liu CG, Ambs S, Cimmino A, Petrocca F Visone R, Iorio M, Roldo C, Ferracin M, et al: A microRNA expression signature of human solid tumors defines cancer gene targets. Proc Natl Acad Sci USA 103: 2257-2261, 2006.

20. Yu P, Wu D, You Y, Sun J, Lu L, Tan J and Bie P: miR-208-3p promotes hepatocellular carcinoma cell proliferation and invasion through regulating ARID2 expression. Exp Cell Res 336 232-241, 2015 .

21. Li H, Zheng D, Zhang B, Liu L, Ou J, Chen W, Xiong S, Gu Y and Yang J: Mir-208 promotes cell proliferation by repressing SOX6 expression in human esophageal squamous cell carcinoma. J Transl Med 12: 196, 2014.

22. Yin K, Liu M, Zhang M, Wang F, Fen M, Liu Z, Yuan Y, Gao S, Yang L, Zhang W, et al: miR-208a-3p suppresses cell apoptosis by targeting PDCD4 in gastric cancer.Oncotarget 7: 67321-67332,2016.

23. Chassagnon G, Bennani S and Revel MP: New TNM classification of non-small cell lung cancer. Rev Pneumol Clin 73: 34-39, 2017 (In French).

24. Lewis BP, Burge CB and Bartel DP: Conserved seed pairing, often flanked by adenosines, indicates that thousands of human genes are microRNA targets. Cell 120: 15-20, 2005.

25. Enright AJ, John B, Gaul U, Tuschl T, Sander C and Marks DS MicroRNA targets in Drosophila. Genome Biol 5: R1, 2003.
26. Cao M, Hou D, Liang H, Gong F, Wang Y, Yan X, Jiang X, Wang C, Zhang J, Zen K, et al: miR-150 promotes the proliferation and migration of lung cancer cells by targeting SRC kinase signalling inhibitor 1. Eur J Cancer 50: 1013-1024, 2014.

27. Ye L, Wang $\mathrm{H}$ and Liu B: miR-211 promotes non-small cell lung cancer proliferation by targeting SRCIN1. Tumour Biol 37: 1151-1157, 2016.

28. Gao Y, Xue Q, Wang D, Du M, Zhang Y and Gao S: miR-873 induces lung adenocarcinoma cell proliferation and migration by targeting SRCIN1. Am J Transl Res 7: 2519-2526, 2015.

29. Cao Y, Zhao D, Li P, Wang L, Qiao B, Qin X, Li L and Wang Y: MicroRNA-181a-5p impedes IL-17-induced nonsmall cell lung cancer proliferation and migration through targeting VCAM-1. Cell Physiol Biochem 42: 346-356, 2017.

30. Wang H, Zhan Y, Jin J, Zhang C and Li W: MicroRNA-15b promotes proliferation and invasion of non-small cell lung carcinoma cells by directly targeting TIMP2. Oncol Rep 37: 3305-3312, 2017.

31. Liu A, Shao C, Jin G, Liu R, Hao J, Song B, Ouyang L and $\mathrm{Hu}$ X: miR-208-induced epithelial to mesenchymal transition of pancreatic cancer cells promotes cell metastasis and invasion. Cell Biochem Biophys 69: 341-346, 2014.

32. Di Stefano P, Cabodi S, Boeri Erba E, Margaria V, Bergatto E, Giuffrida MG, Silengo L, Tarone G, Turco E and Defilippi P: P130Cas-associated protein (p140Cap) as a new tyrosine-phosphorylated protein involved in cell spreading. Mol Biol Cell 15: 787-800, 2004

33. Chen R, Liao JY, Huang J, Chen WL, Ma XJ and Luo XD: Downregulation of SRC kinase signaling inhibitor 1 (SRCIN1) expression by MicroRNA-32 promotes proliferation and epithelial-mesenchymal transition in human liver cancer cells. Oncol Res 26: 573-579, 2018

34. Chen B, Pan W, Lin X, Hu Z, Jin Y, Chen H, Ma G, Qiu Y, Chang L, Hua C, et al: MicroRNA-346 functions as an oncogene in cutaneous squamous cell carcinoma. Tumour Biol 37: 2765-2771, 2016.

35. Yang F, Luo LJ, Zhang L, Wang DD, Yang SJ, Ding L, Li J, Chen D, Ma R, Wu JZ and Tang JH: MiR-346 promotes the biological function of breast cancer cells by targeting SRCIN1 and reduces chemosensitivity to docetaxel. Gene 600: 21-28, 2017.

36. Wang P, Wang H, Li X, Liu Y, Zhao C and Zhu D: SRCIN1 suppressed osteosarcoma cell proliferation and invasion. PLoS One 11: e0155518, 2016.

37. Wang $\mathrm{H}, \mathrm{Wu} \mathrm{C}$, Wan $\mathrm{S}$, Zhang $\mathrm{H}$, Zhou $\mathrm{S}$ and Liu G: Shikonin attenuates lung cancer cell adhesion to extracellular matrix and metastasis by inhibiting integrin $\beta 1$ expression and the ERK1/2 signaling pathway. Toxicology 308: 104-112, 2013.

38. Qi M, Tian Y, Li W, Li D, Zhao T, Yang Y, Li Q, Chen S, Yang Y, Zhang Z, et al: ERK inhibition represses gefitinib resistance in non-small cell lung cancer cells. Oncotarget 9: 12020-12034, 2018.

39. Cao Q, Mao ZD, Shi YJ, Chen Y, Sun Y, Zhang Q, Song L and Peng LP: MicroRNA-7 inhibits cell proliferation, migration and invasion in human non-small cell lung cancer cells by targeting FAK through ERK/MAPK signaling pathway. Oncotarget 7: 77468-77481, 2016.

40. Zhang L, Lin J, Ye Y, Oba T, Gentile E, Lian J, Wang J, Zhao Y, Gu J, Wistuba II, et al: Serum MicroRNA-150 predicts prognosis for early-stage non-small cell lung cancer and promotes tumor cell proliferation by targeting tumor suppressor gene SRCIN1. Clin Pharmacol Ther 103: 1061-1073, 2018.

This work is licensed under a Creative Commons Attribution-NonCommercial-NoDerivatives 4.0 International (CC BY-NC-ND 4.0) License. 